\title{
Evolutionary entropy predicts the outcome of selection: Competition for resources that vary in abundance and diversity
}

\author{
Lloyd Demetrius $^{\mathrm{a}, \mathrm{b}, *}$, Stéphane Legendre ${ }^{\mathrm{c}}$ \\ a Department of Organismic and Evolutionary Biology, Harvard University, Cambridge, Mass., USA \\ ${ }^{\mathrm{b}}$ Max Planck Institute for Molecular Genetics, 14195, Berlin, Germany \\ ${ }^{\mathrm{c}}$ Laboratoire Fonctionnement et Evolution des Systèmes Ecologiques (UMR 7625), Ecole Normale Supérieure, 46 rue d'Ulm, 75230 Paris cedex 05, France
}

\section{A R T I C L E I N F O}

Article history:

Received 24 November 2011

Available online 29 October 2012

\section{Keywords:}

Entropic selection principle

Life-history

Reproductive potential

Demographic variance

\begin{abstract}
A B S T R A C T
Competition between individuals for resources which are limited and diverse in composition is the ultimate driving force of evolution. Classical studies of this event contend that the outcome is a deterministic process predicted by the growth rate of the competing types-a tenet called the Malthusian selection principle. Recent studies of competition indicate that the dynamics of selection is a stochastic process, regulated by the population size, the abundance and diversity of the resource, and predicted by evolutionary entropy-a statistical parameter which characterizes the rate at which the population returns to the steady state condition after a random endogenous or exogenous perturbation. This tenet, which we will call the entropic selection principle entails the following relations:
\end{abstract}

(a) When resources are constant, limited and diverse, variants with higher entropy will have a selective advantage and increase in frequency.

(b) When resources undergo large variations in abundance and are singular, variants with lower entropy will have a selective advantage and increase in frequency.

This article delineates the analytic, computational and empirical support for this tenet. We show moreover that the Malthusian selection principle, a cornerstone of classical evolutionary genetics, is the limit, as population size and resource abundance tends to infinity of the entropic selection principle. The Malthusian tenet is an approximation to the entropic selection principle-an approximation whose validity increases with increasing population size and increasing resource abundance. Evolutionary entropy is a generic concept that characterizes the interaction dynamics of metabolic entities at several levels of biological organization: cellular, organismic and ecological. Accordingly, the entropic selection principle represents a general rule for explaining the processes of adaptation and evolution at each of these levels.

(C) 2012 Elsevier Inc. All rights reserved.

\section{Introduction}

Darwin's evolutionary model of variation, heredity and selection, clearly distinguished between the processes which occur on a generational time scale - the cooperation between parental alleles in an organism, and the processes which unfold on an ecological time scale - the competition between variant types in the population for the available resources.

Mendel's laws of particulate inheritance, proposed in 1860 , have completely resolved the problem of allelic cooperation. The

\footnotetext{
* Corresponding author at: Department of Organismic and Evolutionary Biology, Harvard University, Cambridge, Mass., USA.

E-mail addresses: ldemetr@oeb.harvard.edu, ldemetr@fas.harvard.edu (L. Demetrius), legendre@ens.fr (S. Legendre).
}

laws refuted the dominant model based on the fusion or the blending of parental heritabilities and was finally accepted almost 80 years after its promulgation when it was established that the rules of blending inheritance - the intuitively plausible tenet was essentially a limiting case of the Mendelian rules. Formally, the theory of blending inheritance is the limit $N \rightarrow \infty$, where $N$ denotes the number of alleles governing a trait, of the laws of particulate inheritance (Fisher, 1930). Accordingly, the rules derived from the blending theory are an approximation to the Mendelian laws, an approximation whose validity increases as the number of alleles determining a trait increases.

The blending theory was largely derived from the observation that in phenotypic characters such as size, offspring are generally intermediate between their parents. This empirical fact which was familiar to most naturalists in Darwin's time was sufficiently convincing to discredit alternative models such as the theory of particulate inheritance. Although the rediscovery of Mendel's laws by 
de Vries and Correns in 1900 was a sharp refutation of the blending theory, the particulate model was only accepted after Fisher showed analytically that blending inheritance is an approximation of Mendel's atomic model of genotypic transmission.

The problem of competition between genotypes - the ecological complement of allelic cooperation - also bore Fisher's signature. Fisher realized that any analytic model of the Darwinian argument required both a quantitative depiction of the principle of hereditythe problem which Mendel had resolved, and a mathematical characterization of the principle of selection.

The selection principle Fisher proposed was essentially a formalization of Darwin's notion of the 'struggle for survival', an idea derived from his reading of Malthus' work on populations.

The Fisherian model in its modern incarnation considers competition between a large structured population of effectively infinite size (the incumbent), and a variant population of small size (the invader). The incumbent is assumed to be at demographic equilibrium, that is the distribution of states amongst population members is invariant over time. At demographic equilibrium, the total population size will increase at a rate - the intrinsic rate of increase or Malthusian parameter - which is a function of the individual birth and death rates of the population members.

The model assumes that difference between the demographic properties of incumbent and invader are small and resource abundance is unlimited. The outcome of competition can be characterized in terms of what is now called as:

The Malthusian selection principle. The outcome of selection is a deterministic process predicted by the relative intrinsic rate of increase of invader and incumbent.

According to this principle, the selective advantage of a type, denoted by $s$, is given by

$s=\Delta r$.

Here $\Delta r=r^{*}-r$, where $r$ and $r^{*}$ are the intrinsic rate of increase of the incumbent and the variant types respectively.

The Malthusian selection principle has become a mainstay in evolutionary genetics and evolutionary ecology, Roff (1992), Stearns (1992), Charlesworth (1994) and Rand et al. (1994). The Malthusian parameter and its various surrogates, such as the net-reproduction rate, the basic reproduction number, and the expected number of adult offspring, constitute the decisive and organizing variable in several evolutionary contexts.

Populations may be structured in terms of state variables such as age, size or behavioral traits. Accordingly the evolutionary contexts to which the Malthusian principle pertains cover a large spectrum: the evolution of senescence, Hamilton (1966) and Charlesworth (1994); the spread of pathogens, May et al. (2001) and Fernebro et al. (2008); the spread of altruism and the evolution of cooperation, Hamilton (1964), Rousset and Ronce (2004) and Lehmann and Keller (2006).

The analytic study of natural selection in these evolutionary contexts often ignores factors such as the heterogeneity of the environment, and the impact of this factor on competition between the incumbent and the variant types in a population. The environment was defined by a homogeneous inert resource, and the organisms as active agents appropriating and investing resources into net-offspring production.

Malthusian selection was determined uniquely by the efficiency of the organism to transform a homogeneous inert resource into maintenance and reproduction. Accordingly, the capacity of the organism to acquire resources was never considered a critical determinant of selective outcome.

Lewontin (1982) underscored the limitation of the organism-environment dichotomy which these Malthusian models implicitly invoked by specifying an intrinsic reciprocity of the organism-environment interaction. This reciprocity entails that organisms select and modify the environment they inhabit, whereas the environment responds by imposing constraints on the development and expression of the different genotypes.

According to this organism-environment dichotomy, the selective advantage of an organism, that is the capacity to contribute to the ancestry of future generations, will depend on both its capacity to appropriate resources from an environment which may be highly heterogeneous, and its efficiency in transforming these acquired resources into demographic components which may show large temporal variation in the size or age at which individuals reproduce and die.

This article integrates this conceptual framework in a new class of quantitative models that study competition between related populations under various classes of ecological constraints. We formalize the reciprocity of the organism-environment interaction by considering both the heterogeneity of resources, the variation in their abundance, and the interaction of these factors on populations which may be heterogeneous in age, size and metabolic condition.

Our analysis is based on the ergodic theory of dynamical systems. This mathematical formalism, which has its roots in the theory of stochastic processes and non-equilibrium statistical mechanics, was originally introduced in evolutionary biology to analyze the dynamics of selection in age-structured populations, Demetrius (1974). The formalism has been extended to analyze evolutionary processes in molecular, cellular and ecological networks, Demetrius (1997) and Demetrius and Gundlach (1999). One of the main results which has emerged from this class of models is the analytical fact that robustness, the rate at which a population returns to the steady state condition after a random perturbation, can be characterized by a statistical parameter, called evolutionary entropy, a measure of the diversity of the pathways of energy flow between members of the population. Demographic systems consisting of organisms who reproduce at the same time and at a single stage in their life-history - semelparous organisms - have zero entropy. Populations made up of organisms who reproduce at several distinct stages in their life cycle - iteroparous organisms - have positive entropy, Demetrius (1974).

In this article we will integrate the ergodic theory of dynamical systems and the theory of diffusion processes to analyze the dynamics of invasion in environments where resources vary in abundance and diversity. The models proposed in this paper apply to cellular, demographic and ecological systems, as the analysis in Demetrius et al. (2004) imply. However, the macroscopic parameters that are derived from the ergodic theory analysis do not always admit tractable analytic expressions in studies of cellular and ecological models, and in demographic models where the state variable is given in terms of size. Hence, to simplify the computational aspect of the theory and to emphasize the more conceptual elements, we will restrict our exposition to demographic models and assume that the state variables are described in terms of age.

We assume throughout that the resource is structured and that the interaction between the organism and the environment is reciprocal. We will impose the following conditions on the incumbent and invader population:

(a) The incumbent population is large and of finite size, the invader population is of small size.

(b) Differences in the demographic characteristics of incumbent and invader are small.

(c) The incumbent population is genetically homogeneous.

When the condition of demographic equilibrium prevails, the invasion process will be determined by total population size, whose dynamics is described in terms of the dominant and subdominant eigenvalues of the matrix that characterizes the equilibrium state. 
In view of the reciprocity of the organism-environment interaction, demographic equilibrium will rarely be attained, hence the classical invasion methods are no longer pertinent in analyzing the competition dynamics. The problem of invasion in systems whose steady states are non-equilibrium configurations was resolved by invoking the ergodic theory of dynamical systems to characterize the steady states in terms of a new phase space, the space of genealogies, Demetrius (1997). The invasion process will be regulated by the number of typical genealogies, called the effective population size. The asymptotic rate of increase of the effective size is described by evolutionary entropy.

We will show that the outcome of competition between incumbent and invader can be characterized by what we call:

The entropic selection principle: The outcome of selection is a stochastic process predicted by evolutionary entropy and is contingent on environmental variables, the resource abundance and the resource diversity.

According to the entropic selection principle, evolutionary entropy determines the invasion exponent, and the selective advantage is now given by

$s=-\left(\phi-\frac{\gamma}{M}\right) \Delta S$.

Here $\Delta S=S^{*}-S$, where $S$ and $S^{*}$ denote the entropy of the incumbent and variant type.

The quantity $\phi$ and $\gamma$ are functions of the individual birth and death rates. They are defined as the first and the third moments of a random function defined on the space of genealogies. We will show that these two demographic variables are correlated with environmental or resource parameters. The quantity $\phi$ is called the reproductive potential. It is correlated with variations in the resource abundance: $\phi<0$ corresponds to a constant resource abundance, $\phi>0$ a variable resource abundance. The quantity $\gamma$ is called the demographic index and characterizes the degree of heterogeneity of the resource: $\gamma<0$ describes low heterogeneity - a singular resource, $\gamma>0$ describes high heterogeneity - a diverse resource.

The outcome of selection, as described by Eq. (2) can be qualitatively described as follows:

(a) When resource abundance is constant and diverse, variants with higher entropy have a selective advantage and will increase in frequency.

(b) When resource varies in abundance and is singular, variants with lower entropy have a selective advantage and will increase in frequency.

The Malthusian selection principle as formalized by Eq. (1), is expressed uniquely in terms of the asymptotic growth rate. This is the rate of increase of total population size at demographic equilibrium. The model assumes that resource is unlimited and population size large, effectively infinite.

The entropic selection principle, by contrast, is formulated in terms of entropy. We will show that the Malthusian tenet is a limiting case of the entropic selection principle. The relation between the two tenets can be formally annotated as follows:

The Malthusian selection principle is the limit, as $M \rightarrow \infty, R \rightarrow$

$\infty$, where $M$ denotes population size and $R$ denotes resource abundance, of the entropic selection principle.

Accordingly, the Malthusian principle is an approximation to the entropic selection principle-an approximation whose validity increases with increasing population size and increasing resource abundance.

Earlier studies of the invasion process, Demetrius (1997), imposed conditions of finite size and resource abundance, but ignored the effects of resource diversity. The extensions of the

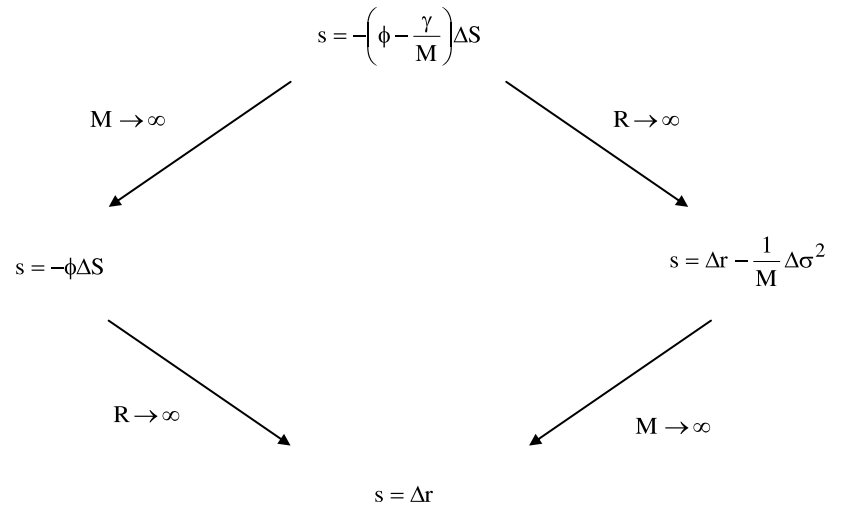

Fig. 1. Relation between resource abundance $R$, population size $M$ and selective advantage $s$.

Malthusian selection principle to incorporate resource constraints and the effect of size can be expressed in the following terms:

When population size is finite and resource abundance is unlimited, the measure of selective advantage is given by

$s=\Delta r-\frac{1}{M} \Delta \sigma^{2}$

Here $r$ and $\sigma^{2}$ denote the intrinsic rate of increase and the demographic variance.

Furthermore, as can be observed from Eq. (2), when resource abundance is limited and population size is infinite, the index of selective advantage becomes

$s=-\phi \Delta S$.

Computational and empirical studies in support of (3) and (4) are described in Kowald and Demetrius (2005) and Ziehe and Demetrius (2005). The relation between the four measures of selective advantage is described in Fig. 1.

The blending theory of inheritance and the Malthusian selection principle are tenets which have played a critical role in evolutionary genetics. Both theories are valid under certain limiting conditions, the first when the number of alleles is large, effectively infinite; the second when resource abundance $R$, and population size $M$ are both infinite.

The blending theory owes its 80 year influence to the fact that it was consistent with the inheritance of quantitative traits. The theory was only rejected when it was established that blending inheritance is simply a degenerate case of Mendel's particulate model.

The Malthusian principle of selection currently represents the dominant paradigm in studies of the evolution of life-history. The principle also undergirds epidemiological studies through the notion of $R_{0}$, the net-reproduction rate, and is a critical parameter in studies of the evolution of cooperation through the concept of inclusive fitness.

The dominance of the Malthusian paradigm emanates partly from the observation that the in vitro growth rate of a population often predicts its invasibility in in vivo conditions. The fact that the Malthusian model is a limiting case of the entropic selection principle, however, suggests that the apparent predictive power of the Malthusian principle derives primarily from the fact that both population size and resource abundance are large, effectively infinite, in the experimental conditions which seem to be consistent with the Malthusian tenet.

\section{Historical overview}

The two most central elements of the neo-Darwinian argument are the principle of inheritance and the principle of selection. 
Mendel's discovery of the quantitative rules relating genotype with phenotype resolved the problem of inheritance. The integration of Mendel's theory in evolutionary genetics in 1920 provided an incentive for evolutionary theorists to elucidate the laws of selection, and thereby develop a coherent framework for a quantitative model of Darwin's theory.

Wright (1945) addressed the problem of selection in a series of models which studied competition between related types in a population. These models of selection ignored factors such as age, fecundity and population size, and focused uniquely on differences in viability. Viability selection models now form the main core of classical population genetics. The main results of these models are described in texts such as Crow and Kimura (1970) and Ewens (2004). Kimura has built on the earlier studies of Wright by systematically integrating the mathematical formalism of diffusion processes in models of population genetics. Extensions of these models by Gillespie (1974) have been very influential in studies of genetics models of finite size.

The Wright-Kimura-Gillespie models introduced a stochastic component in population genetics. However, the analysis remains embedded within the framework of classical viability selection and ignored both the demographic structure of populations and the effect of resource abundance and resource diversity on the dynamics of selection.

The integration of demographic structure in studies of selection is primarily due to Fisher (1930), where the notion of Malthusian parameter was first introduced. The invasion dynamics of structured populations were later developed in studies by Charlesworth and Williamson (1975), Pollack and Kempthorne (1970), Metz et al. (1992) and Houston and McNamara (1999), among others. Although these studies discussed the effects of resource abundance on the dynamics of selection, this aspect was first systematically considered by MacArthur (1962). The MacArthur models however ignored demographic structure.

The $r-K$ models of selection which were essentially based on Mac Arthur's analytic studies of selection in density dependent populations, represented the effect of the environment on the population dynamics uniquely in terms of its carrying capacity $K$. The heterogeneity of the environment, the demographic structure of the population and the reciprocity of the organism-environment interaction played no role in these studies. The limitations of the $r-K$ class of models have been emphasized through empirical studies of the invasion process, Lawton and Brown (1986). These studies show that the amplitude of population fluctuations is often the critical determinant in predicting the outcome of invasion.

These observations have led to the study of more complex models of the invasion process, see for example Kisdi and Geritz (1999), Dieckmann and Ferrière (2004), Leturque and Rousset (2002) and Rousset and Ronce (2004). The studies concern structured populations in which individual fecundity and mortality rates depend on population size and environmental variables, and in studies of cooperation, the parameter relatedness.

The discrete time models of these invasion processes parameterize individuals in terms of their age, size or some behavioral trait. The systems are assumed to be in demographic equilibrium. In this case, the dominant eigenvalue of the process determining the steady state behavior represents the critical parameter. This quantity describes the invasion exponent, the rate of increase of the total population size.

The conditions of demographic equilibrium do not in general prevail in systems which involve interactions between a population and an environment whose resources may be heterogeneous in composition and variable in abundance. The organism-environment dichotomy, as articulated in Lewontin (1982), emphasized the reciprocal nature of this interaction. This reciprocity entails that at steady state, population processes may be far from demographic equilibrium; consequently characterizing the invasion exponent in terms of the eigenvalues of the relevant matrices may not provide a valid predictor of the outcome of competition.

Demetrius (1974), in studies of age-structured population models, showed that the non-equilibrium steady state of population processes can be analyzed by appealing to the ergodic theory of dynamical systems. In these models, the phase space described by an age distribution is replaced by a phase space consisting of genealogies-a recording of the successive ancestors of a particular individual which at time zero is in the first age group. The state of the population is an invariant probability measure on the configuration space of genealogies. In the ergodic theory formalism, the dynamical entropy of the probability measure defined on the space of genealogies is now the critical parameter. This entropy measure, called evolutionary entropy, is the rate of increase of the 'typical' number of genealogies generated by the population process.

The ergodic theory formalism was adduced to derive a new class of population variables - reproductive potential, demographic index - to analyze the non equilibrium behavior of population processes. The formalism is also applicable to structured populations parameterized in terms of morphometric or behavioral states, Demetrius et al. (2004).

Ergodic theory was later integrated with the theory of diffusion processes to study the invasion dynamics of structured population of finite size, Demetrius (1997), Demetrius and Gundlach (1999) and Demetrius et al. (2004). The analysis described in this paper is the natural extension of the ergodic theory-diffusion process methods to study competition in organism-environmental interactions where resources may vary in abundance and diversity.

\section{Structured populations: macroscopic parameters and micro- scopic dynamics}

All natural populations manifest a variability in the age at which individuals reproduce and die. This heterogeneity has its origin in the molecular processes that encode an individual's ontogeny. In populations of cells, the heterogeneity is a consequence of the randomized distribution of metabolic components - proteins and other molecules - which occurs when the cell divides. This means that a clone of cells consisting of genetically identical individuals will show a variability in their demographic and physiological properties. The individual cells in the clone will differ in terms of their metabolic constituents and size, and hence the clone will exhibit a variability in the rate at which the individuals traverse the different stages of the cell cycle.

In multicellular organisms, heterogeneity is derived from the random deviations of the embryonic process from its prescribed ontogenic path. Hence demographic variability is not just a property of cellular systems and primitive organisms. It is also observed in human populations as indicated by the phenotypic differences observed in genetically identical twins.

These observations entail that demographic heterogeneity is a universal property of all metabolic replicating entities. Hence any genetically homogeneous population will be characterized by a variability in the developmental state at which individuals reproduce and die (Schmalhausen, 1949). Heterogeneity in survivorship and fecundity is thus an intrinsic feature of all replicating entities. This variability in life cycle is highly related to the adaptive properties of the population, that is to its persistence and to the capacity of individual organisms to appropriate resources from the environment and to transform the resources into metabolic work and net-offspring production. The analytical characterization of this life-cycle variability is thus critical in any study of the adaptive and evolutionary dynamics of populations. 


\subsection{Discrete and continuous models}

We consider a population in which the state of the individuals are parameterized in terms of some demographic or physiological state. Let $u_{i}(t)$ denote the number of individuals in state $i$ at time $t$, where $1 \leq i \leq d$.

The change in the distribution $\tilde{u}(t)=\left[u_{1}(t), \ldots, u_{d}(t)\right]$ can be described by the discrete dynamical system

$\tilde{u}(t+1)=A(t) \tilde{u}(t)$.

Here $A(t)=\left\{a_{i j}(t)\right\}$, where $a_{i j}(t) \geq 0$. The elements $a_{i j}(t)$ are the individual transition rates between state $i$ and state $j$.

The analysis in this paper will be restricted to models in which the individuals are parameterized by their age and the transitions $a_{i j}(t)$ denote age-specific birth and death rates. In this case the matrix $A(t)$ is a Leslie matrix. The first row in the matrix describes the age-specific birth rates, and the off-diagonal terms the age-specific survivorship rates. The elements $b_{i}(t)$ denote the probability that an individual in age class $i$ survives to age class $i+1$. The quantities $m_{i}(t)$ denote the mean number of offspring produced by individuals in age class $i$.

The continuous analogue of Eq. (5) is specified by considering the function $u(x, t)$, the number of individuals of age $x$ at time $t$. The population size $N(t)$ is now given by

$N(t)=\int_{0}^{\infty} u(x, t) d t$

The changes in the age distribution $u(x, t)$ are determined by the age-specific death rate $\mu(x, N)$, and the age-specific birth rate $m(x, N)$ which both depend on population size. The dynamics $u(x, t)$ is given by

$\frac{\partial u}{\partial x}+\frac{\partial u}{\partial t}=-\mu(x, N) u(x, t)$,

$u(0, t)=\int_{0}^{\infty} u(x, t) m(x, N) d x$

We assume that the system has attained a steady state where the population is described by an age-specific death rate $\mu(x)$ and an age-specific birth rate $m(x)$.

Let $l(x)$ denote the age-specific survivorship function, that is the probability that an individual born at age zero survives to age $x$. The net-reproductive function $V(x)$ is given by

$V(x)=l(x) m(x)$.

The function $V(x)$ represents the individual or the microscopic parameters that define the population process. The ergodic theory of dynamical systems and the formalism of statistical mechanics can be used to generate a class of macroscopic variables from the net-reproductive function $V(x)$. These macroscopic variables are essentially statistical averages generated by a probability distribution defined in terms of $V(x)$. This perspective gives a comprehensive approach towards the evolutionary entropy concept, and the derivation of standard population variables, such as population growth rate and generation time.

\subsubsection{Evolutionary entropy}

Evolutionary entropy $S$ is given by

$S=-E[\log p(X)]$,

where $X$ is the random variable associated with a distribution $p(x)$ defined in terms of the net-reproductive function $V(x)$. Hence,

$S=-\int_{0}^{\infty} p(x) \log p(x) d x$

Two classes of distributions can be characterized. One is given by considering the net-reproduction rate, $R_{0}$ defined by
$R_{0}=\int_{0}^{\infty} V(x) d x$

The distribution in this case is given by $p(x)=\frac{V(x)}{R_{0}}$.

The second distribution is obtained by considering the population growth rate parameter $r$, which is the unique real root of the equation

$1=\int_{0}^{\infty} \exp (-r x) V(x) d x$

The distribution in this case is given by $p(x)=\exp (-r x) V(x)$.

It is a well known fact that the net-reproduction rate $R_{0}$ and the population growth rate $r$ are related. We have

$R_{0}>1 \Leftrightarrow r>0$,

$R_{0}=1 \Leftrightarrow r=0$,

$R_{0}<1 \Leftrightarrow r<0$.

In this article we will deal uniquely with the distribution $p(x)=$ $\exp (-r x) V(x)$. The quantity $p(x)$ describes the probability that the mother of a randomly chosen newborn belongs to age class $(x, x+d x)$. Hence the entropy $S$ represents the uncertainty in the age of the mother of a randomly chosen newborn.

Fig. 2(A) describes the net-reproductive function of an annual plant (low entropy) where reproduction is concentrated at a single stage of the life cycle, and a perennial plant (high entropy) where reproduction occurs at several distinct stages of the life cycle.

Consider the expectation $T=E(X)$ given by

$T=\int_{0}^{\infty} x p(x) d x$

The parameter $T$ describes the mean age of mothers at the birth of their offspring, and is called generation time. We can now use $T$ to define the entropy rate

$H=\frac{S}{T}=-\frac{\int p(x) \log p(x) d x}{\int x p(x) d x}$.

The entropy rate $H$ is positively correlated with the rate at which the population returns to its steady state condition after a random perturbation in the individual birth and death rates (Demetrius et al., 2004). Hence evolutionary entropy describes the robustness of the population, that is its ability to maintain a stable trajectory in the face of random perturbations in the age-specific birth and death rates.

\subsubsection{Demographic variables: macroscopic parameters of population dynamics}

The population growth rate $r$ is implicitly defined in terms of the function $V(x)$ by the integral equation (6). The statistical mechanics formalism, described in Demetrius (1983), provides an algorithm for generating macroscopic variables from the function $V(x)$ and the population growth rate $r$.

Let $r(\delta)$ denote the growth rate associated with the netreproductive function $V(x)^{1+\delta}$. A Taylor expansion of $r(\delta)$, see Demetrius (1997), yields

$r(\delta)=r(0)+\delta r^{\prime}(0)+\frac{\delta^{2}}{2 !} r^{\prime \prime}(0)+\frac{\delta^{3}}{3 !} r^{\prime \prime \prime}(0)+\cdots$

where $r^{\prime}(0)=\phi, r^{\prime \prime}(0)=\sigma^{2}, r^{\prime \prime \prime}(0)=\kappa$. The quantities $\phi, \sigma^{2}$ and $\kappa$ are called the reproductive potential, the demographic variance and the correlation index, respectively.

We have, Demetrius et al. (2004), derived analytical expressions for these quantities: 

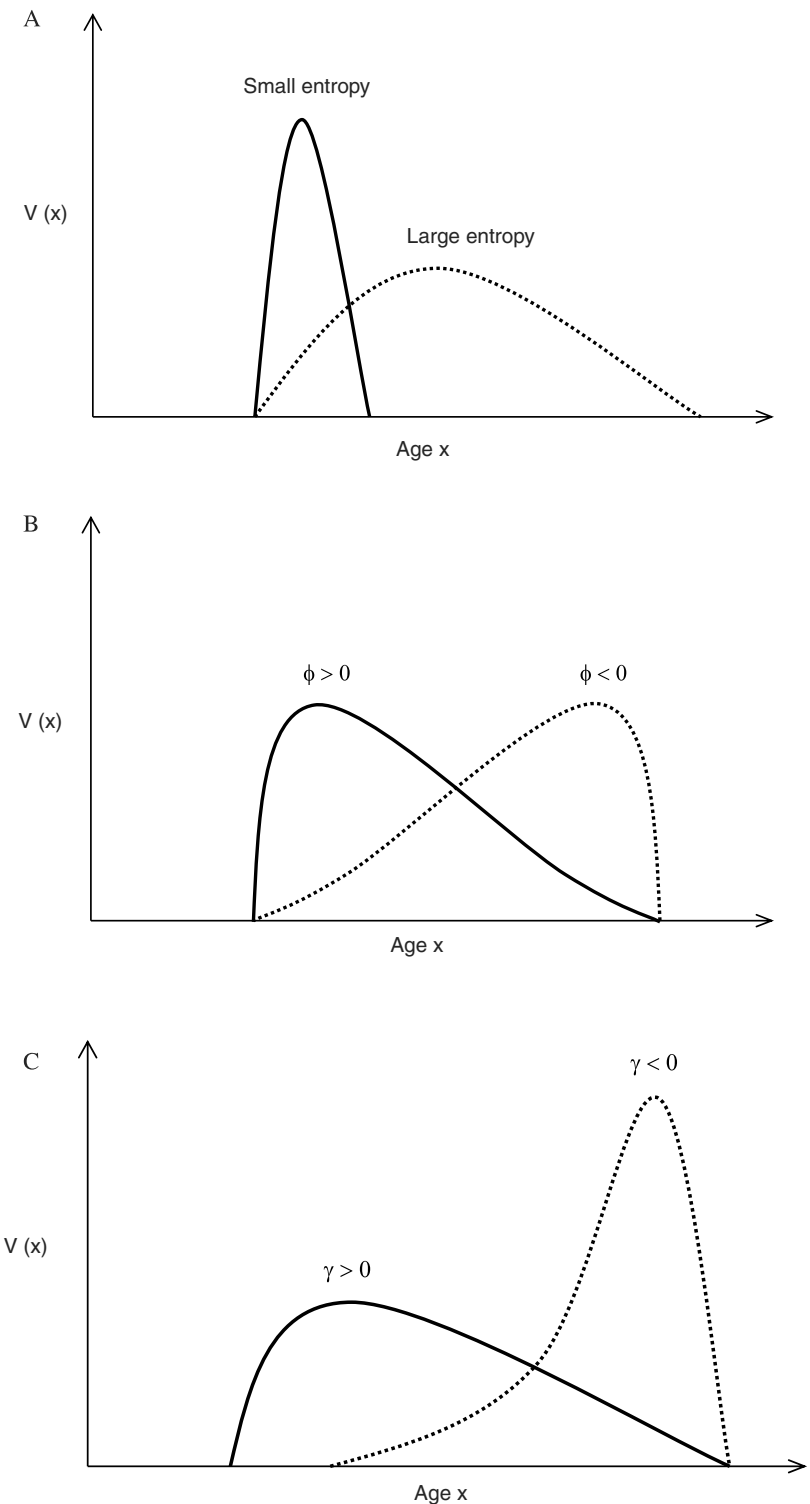

Fig. 2. Schematic representation of the net-reproductive function $V(x)$ corresponding to (A) small and large entropy, (B) the reproductive potential $\phi>0$ and $\phi<0$, (C) the demographic index $\gamma>0$ and $\gamma<0$.

$\phi=\frac{1}{T} \int_{0}^{\infty} p(x) \log V(x) d x$,

$\sigma^{2}=-\frac{1}{T} \int_{0}^{\infty} p(x) W^{2}(x) d x$

$\kappa=\frac{1}{T} \int_{0}^{\infty} p(x) W^{3}(x) d x-\frac{\sigma^{2}}{T} \int_{0}^{\infty} x p(x) W(x) d x$,

where

$W(x)=-x \phi+\log V(x)$.

The functions $\phi, \sigma^{2}$ and $\kappa$ are the first, second and third moments of the random variable $\log V(x)$ with respect to the probability distribution $p(x)$.

The directional derivatives

$\left.\frac{d r}{d \delta}\right|_{\delta=0}$ and $\left.\frac{d \sigma^{2}}{d \delta}\right|_{\delta=0}$

describe changes in the growth rate $r$ and the variance $\sigma^{2}$. These changes are induced by a perturbation in the net-reproductive function $V(x)$ to yield a function $V(x)^{1+\delta}$. The quantities $r(\delta)$ and $\sigma^{2}(\delta)$ are the growth rate and variance parameters associated with the function $V(x)^{1+\delta}$. The expressions given by (11) can be explicitly computed. We have

$\left.\frac{d r}{d \delta}\right|_{\delta=0}=\frac{1}{T} \int_{0}^{\infty} p(x) \log V(x) d x$.

This expression is precisely $\phi$, the reproductive potential. The reproductive potential can therefore be considered as the sensitivity of the growth rate parameter $r$ to global perturbations in the netreproductive function.

By considering the demographic variance function given by Eq. (9) we obtain

$$
\begin{aligned}
\left.\frac{\partial \sigma^{2}}{\partial \delta}\right|_{\delta=0}= & -\frac{2}{T} \int_{0}^{\infty} p(x) W^{2}(x) d x+\frac{1}{T} \int_{0}^{\infty} p(x) W^{3}(x) d x \\
& -\frac{\sigma^{2}}{T} \int_{0}^{\infty} x p(x) W(x) d x .
\end{aligned}
$$

This expression is $\gamma$, the demographic index. Hence,

$\left.\frac{d r}{d \delta}\right|_{\delta=0}=\phi$ and $\left.\frac{d \sigma^{2}}{d \delta}\right|_{\delta=0}=\gamma$.

We can infer from Eq. (12), that for $\delta$ small and $\phi \neq 0, \gamma \neq 0$, the following relations hold:

$\Delta r \approx \phi \delta, \quad \Delta \sigma^{2} \approx \gamma \delta$.

The quantities $r, H, \phi, \gamma$ and $\sigma^{2}$ are all functions of the netreproductive function $V(x)$. They constitute macroscopic variables which describe various demographic and adaptive properties of the population. The reproductive potential $\phi$ and the demographic index $\gamma$ have certain geometric properties which we now delineate.

The reproductive potential $\phi$. It is easy to verify from Eqs. (7) and (8) that

$\phi=r-H$.

Hence, we have

$\phi<0 \Rightarrow r<H, \quad \phi>0 \Rightarrow r>H$.

Fig. 2(B) gives a net-reproductive functions $V(x)$ that corresponds to the case $\phi<0$ and $\phi>0$.

We observe from (14) that $\phi<0$ is characteristic of populations whose growth rate is smaller than entropy. Typically, $\phi<0$ will describe populations whose organisms have large body sizelarge mammals and birds. In such populations growth is slow and the robustness or stability of population numbers, as measured by entropy $H$, is strong. The condition $\phi>0$ will be descriptive of populations whose growth rate exceeds the entropy. Typically $\phi>0$ will be representative of populations of small body size, for example insects and small birds. In such populations, growth is always rapid. However, population numbers will be subject to large fluctuations.

The demographic index $\gamma$. It can be shown using (9) and (10) that

$\gamma=2 \sigma^{2}+\kappa$.

Hence,

$\gamma<0 \Rightarrow \sigma^{2}<\frac{\kappa}{2}, \quad \gamma>0 \Rightarrow \sigma^{2}>\frac{\kappa}{2}$.

Since $\sigma^{2}$ measures the dispersion of $V(x)$, we conclude from (17) that $\gamma<0$ corresponds to a peaked distribution for $V(x)$ and $\gamma>0$ a flat distribution. These patterns are described in Fig. 2(C). 
Table 1

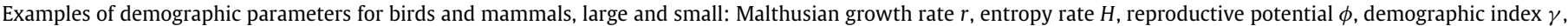
demographic variance $\sigma^{2}$.

\begin{tabular}{|c|c|c|c|c|c|c|c|}
\hline Taxon & Species & Body size (g) & $r$ & $H$ & $\phi$ & $\gamma$ & $\sigma^{2}$ \\
\hline Large mammal & Ovis canadensis & 58000 & 0.037 & 0.336 & -0.230 & 1.099 & 0.083 \\
\hline Small mammal & Tamias striatus & 100 & 0.963 & 0.602 & 0.361 & 0.202 & 0.133 \\
\hline Large bird & Anser caerulescens & 2500 & 0.154 & 0.44 & -0.287 & 0.6982 & 0.058 \\
\hline Small bird & Petronia petronia & 35 & 0.7655 & 0.6523 & 0.113 & 0.2365 & 0.090 \\
\hline
\end{tabular}

The relation given by (16) indicates that the condition $\gamma<$ 0 imposes strong constraints on the geometry of the netreproductive function-namely a highly peaked distribution. Such a function would be typical of organisms whose reproduction is concentrated in a few age classes. Such populations will be described by a low growth rate and a low entropy. The combined effect of these two features entail a high probability of extinction, hence such populations will be rare. The condition $\gamma>0$ entails a net-reproductive function with a broad distribution. This situation will accommodate populations where the growth rate and entropy may assume a large range of values. These observations indicate that the condition $\gamma<0$ will be atypical, whereas $\gamma>0$ will be observed in most natural populations.

Table 1 gives values of $r, H, \phi, \gamma$ and $\sigma^{2}$ for certain species of mammals and birds. We note that $\gamma>0$ for all species and the range of values for $\phi, r$ and $H$ are consistent with the arguments described.

\section{The invasion dynamics in structured populations}

The reciprocal nature of the organism-environment interaction entails that the dynamics of invasion of a variant type in a resident population integrates two related factors:

1. The capacity of the organism to appropriate resources from the external environment.

2. The efficiency with which the organism transforms the acquired resources into energy which is then used for maintenance and reproduction.

The analytic depiction of these two factors requires an understanding of the relation between environmental characteristics, such as resource abundance and resource diversity, and the demographic and phenotypic properties of the organism.

Empirical studies of the organism-environment dichotomy using plant populations, Raunkiær (1934), have shown that the reciprocal nature of the interaction entails certain classes of correlations between environmental properties and phenotypic traits. Levins and Lewontin (1985, Chapter 4) expressed this correlation as a general principle. These relations can be expressed as follows:

If organisms respond to their environments, then the environment may be read through the organism and units of environmental measurements can be translated into units of phenotype.

We will furnish analytic and empirical support for a similar tenet which relates environmental measures - resource abundance and resource diversity - with demographic parameters reproductive potential and the demographic index. This organism-environment tenet will provide a framework for a model of the dynamics of selection which we will adduce to derive the entropic principle of selection.

\subsection{The organism-environment interaction}

The studies of population dynamics as described in Demetrius (1997) indicate that the steady state dynamics of structured populations can be characterized in terms of the population growth rate $r$ and the demographic variance $\sigma^{2}$. Accordingly, if $N(t)$ denotes the population size when steady state is attained, the change in $N(t)$ will be given by the stochastic differential equation

$d N(t)=r N(t) d t+\sigma \sqrt{N(t)} d W_{t}$

where $W_{t}$ denotes standard Brownian motion.

We will assume that the steady state dynamics of the resources can also be characterized in terms of a production rate $\alpha$, and a variance $\beta$. Hence, if $R(t)$ is the resource abundance, then the resource dynamics at steady state will be described by

$d R(t)=a R(t) d t+\beta \sqrt{R(t)} d W_{t}$.

The resource abundance and population size are assumed to be coupled by an analytic relation of the form

$N(t)=f(R(t))$.

We will appeal to the reciprocity of the organism-environment interaction to propose an analogue of the correlation between environmental measurements and phenotypic properties discussed earlier.

Our analogue of Raunkiaer's empirical rule is denoted as:

The organism-environment interaction principle:

If the resource process and the population process are in dynamical equilibrium, then the changes in resource abundance and diversity will be positively correlated with the demographic variables, reproductive potential and demographic index, respectively.

We will derive an analytic support for this principle by considering the effects of changes in resource abundance on the growth rate and demographic variance of the population.

Let $r(\delta)$ and $\sigma^{2}(\delta)$ denote the change in growth rate and demographic variance induced by a change $\Delta R$ in resource abundance. Assuming that the resource process and the population process are in dynamic equilibrium, we conclude that, if $\Delta \alpha$ and $\Delta \beta$ denote the changes in resource production rate and resource heterogeneity then we have

$\left.\Delta \alpha \approx \frac{d r}{d \delta}\right|_{\delta=0},\left.\quad \Delta \beta \approx \frac{d \sigma^{2}}{d \delta}\right|_{\delta=0}$.

Since $\left.\frac{d r}{d \delta}\right|_{\delta=0}=\phi$ and $\left.\frac{d \sigma^{2}}{d \delta}\right|_{\delta=0}=\gamma$, we conclude that

$\Delta \alpha \approx \phi, \quad \Delta \beta \approx \gamma$.

The correlation implies a correspondence between the environmental variables, as characterized by resource abundance and resource heterogeneity, and the demographic variables, as defined by the reproductive potential and the demographic index. Empirical support for the correspondence between environmental variables and demographic parameters are discussed in Section 6.2.

The organism-environment interaction principle indicates that when steady state and dynamical equilibrium conditions prevail, environmental measures such as variations in resource abundance and variations in diversity can be inferred from demographic parameters related to the growth rate and the demographic variance. 


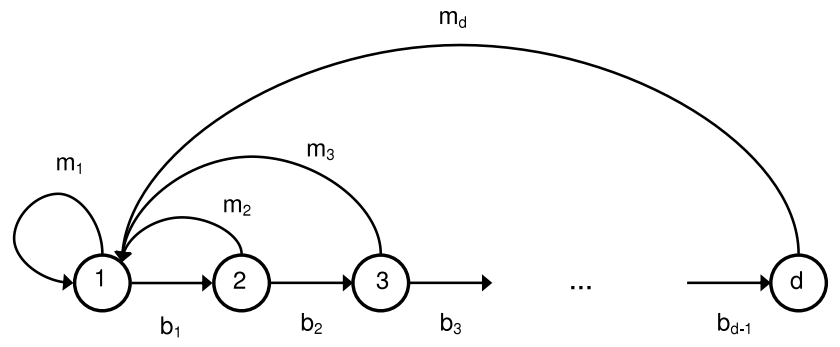

Fig. 3. Representation of the life cycle graph leading to the Leslie matrix formulation.

We have in earlier studies, exploited the theory of diffusion processes to derive measures of selective advantage in studies of competition between an incumbent and a variant type. In these studies, the effect of the action of resource constraints on the dynamics of selection was not made explicit.

In Section 5, we will explicitly introduce the notion of resource constraints and assume that the resource abundance $R(t)$ satisfies a stochastic differential equation of the form (18). We furthermore assume that the resource abundance $R(t)$ and the population size $N(t)$ are coupled by a general relation of the form $N(t)=f(R(t))$.

We will distinguish between competition under conditions of unlimited and limited resources and derive corresponding measures of selective advantage.

1. Resources unlimited. Resource abundance is said to be unlimited when the net-reproductive function $V(x)$ remains invariant in the face of changes $\Delta R$ in the resource abundance. When this condition holds, we will show that the outcome of competition between variant and incumbent is completely determined by the population size $M$ and the quantities $\Delta r$ and $\Delta \sigma^{2}$. The selective advantage will be given by $s=\Delta r-\frac{1}{M} \Delta \sigma^{2}$.

2. Limited Resources. Resource abundance is said to be limited, when the net-reproductive function $V(x)$ undergoes change as a result of a change $\Delta R$ in the resource abundance. When this condition holds, we will show that the outcome of competition between variant and incumbent will be determined by the population size $M$ and the quantity $\Delta S$, the change in entropy rate. The outcome will necessarily be contingent on changes in resource abundance and diversity. According to the organism-environment principle, these changes will be correlated with the demographic variables $\phi$ and $\gamma$, and the selective advantage is $S=-\left(\phi-\frac{\gamma}{M}\right) \Delta S$.

\subsection{Models of competition}

The study of competition we present will be in terms of the discrete Leslie model (see Fig. 3). We assume that the incumbent population $X$ is described in terms of the function $V_{j}$ and the variant population $X^{*}$ in terms of the function $V_{j}^{*}$. Let $r, \sigma^{2}, S$ denote the growth rate, demographic variance and entropy of $X$, and $r^{*}, \sigma^{2^{*}}, S^{*}$ the corresponding macroscopic parameters of $X^{*}$.

In the subsequent analysis we will impose no constraints on the relation between $V_{j}$ and $V_{j}^{*}$. Consequently, the quantities $\Delta r, \Delta \sigma^{2}, \Delta S$ will simply describe the changes in macroscopic parameters induced by the mutation.

Let $N(t)$ and $N^{*}(t)$ denote the population size of the incumbent and the variant, respectively. Our study of the invasibility of the variant $X^{*}$ will depend on the analysis of the stochastic dynamics of the frequency

$p(t)=\frac{N^{*}(t)}{N(t)+N^{*}(t)}$

in continuous time (due to a diffusion approximation).
The invasion process concerns the interaction between the dynamics of $X^{*}$, which is assumed initially rare, and the dynamics of $X$, which is assumed to be in the neighborhood of the steady state. The model assumes that initially the populations $X$ and $X^{*}$ evolve independently of each other but when certain sizes are attained, interaction between the two types will occur on account of the resource constraints.

Numerical studies discussed in Demetrius et al. (2004) and Demetrius and Ziehe (2007) suggest that convergence to the steady state is much faster than the selection process. We will therefore treat both populations as if they were at steady state. In view of this condition, an inherently non-linear problem can be studied by considering the linearized dynamics of the two interacting types.

Our analysis revolves around the evolution of the probability densities $f(N, t)$ and $f^{*}\left(N^{*}, t\right)$ of the population size $N(t)$ and $N^{*}(t)$. A fundamental aspect of the argument is a central limit theorem in dynamical systems, see Demetrius et al. (2004), which shows that the dynamics of each density function can be described in terms of the population growth rate $r$, and the demographic variance $\sigma^{2}$.

In the case of the resident population of size $N(t)$, the evolution of the density $f(N, t)$ is given by the solution of the Fokker-Planck equation

$\frac{\partial f}{\partial t}=-r \frac{\partial(f N)}{\partial N}+\sigma^{2} \frac{\partial^{2}(f N)}{\partial N^{2}}$.

We obtain a similar representation for the variant population of size $N^{*}(t)$ and its density $f^{*}\left(N^{*}, t\right)$, namely

$\frac{\partial f^{*}}{\partial t}=-r^{*} \frac{\partial\left(f^{*} N^{*}\right)}{\partial N^{*}}+\sigma^{2 *} \frac{\partial^{2}\left(f^{*} N^{*}\right)}{\partial N^{* 2}}$.

Equivalently, we could also represent $N(t)$ and $N^{*}(t)$ as solutions of the stochastic differential equations

$d N=r N d t+\sigma \sqrt{N} d W_{t}$,

$d N^{*}=r^{*} N^{*} d t+\sigma^{*} \sqrt{N^{*}} d W_{t}^{*}$,

where $W_{t}$ and $W_{t}^{*}$ are standard Brownian motions.

Now write $M(t)=N(t)+N^{*}(t)$. Assume that the competition is local and unfolds on a time scale where $M(t)$ is constant. We write $M(t)=M$.

To investigate the outcome of competition between the resident and the variant, we let $\psi(p, t)$ denote the probability density function of the stochastic process which describes the change in frequency $p$. The probability density $\psi$ solves the Fokker-Planck equation

$\frac{\partial \psi}{\partial t}=-\frac{\partial[\alpha(p) \psi]}{\partial p}+\frac{1}{2} \frac{\partial^{2}[\beta(p) \psi]}{\partial p^{2}}$

where

$\alpha(p)=p(1-p)\left[\Delta r-\frac{\Delta \sigma^{2}}{M}\right]$,

$\beta(p)=\frac{p(1-p)}{M}\left[\sigma^{2} p+\sigma^{* 2}(1-p)\right]$.

Here $\Delta x=x^{*}-x$, where $x$ and $x^{*}$ are the demographic parameters describing the resident and invading type.

The problem of extinction-fixation of the variant type can be analyzed by considering the Kolmogorov backward equation

$$
\frac{\partial \psi}{\partial t}=-\alpha(p) \frac{\partial \psi}{\partial p}+\frac{1}{2} \beta(p) \frac{\partial^{2} \psi}{\partial p^{2}}
$$

with the boundary conditions

$\psi(0, t)=1, \quad \psi(1, t)=0$. 
Let $P(y)$ be the probability that the mutant invades the population, where $y$ is the initial frequency of the mutant. We have shown that $P(y)$ is given by

$$
P(y)=\frac{1-\left(1-\frac{\Delta \sigma^{2}}{\Delta \sigma^{2}+\sigma^{2}} y\right)^{\frac{2 M s}{\Delta \sigma^{2}}+1}}{1-\left(1-\frac{\Delta \sigma^{2}}{\Delta \sigma^{2}+\sigma^{2}}\right)^{\frac{2 M s}{\Delta \sigma^{2}}+1}}
$$

where

$s=\Delta r-\frac{1}{M} \Delta \sigma^{2}$.

Except in the degenerate case of $\frac{2 M}{\Delta \sigma^{2}}=-1$, the function $P^{\prime}(y)$ cannot vanish and the concavity of $P(y)$ can be expressed in terms of $s$ only.

As shown in Demetrius and Gundlach (1999), we have by a study of $P(y)$,

$s>0 \Rightarrow P(y)$ convex, $\quad s<0 \Rightarrow P(y)$ concave.

These observations will be adduced to derive two measures of selective advantage.

\subsection{Selective advantage: growth rate and demographic variance}

The function $P(y)$ denotes the probability that a mutant with initial frequency $y$ invades the population. In view of (21), the degree of curvature of $P(y)$ depends on the magnitude of $s$, that is on the value of $\Delta r, \Delta \sigma^{2}$ and $M$. The dependency on $y$ of $P(y)$ has been graphically analyzed in Demetrius et al. (2004). These studies show the following:

(a) $\Delta r>0, \Delta \sigma^{2}<0$ : the mutant invades almost surely.

(b) $\Delta r<0, \Delta \sigma^{2}>0$ : the mutant becomes extinct almost surely.

(c) $\Delta r>0, \Delta \sigma^{2}>0$ :

(i) $M>\frac{\Delta \sigma^{2}}{\Delta r}$, the mutant invades almost surely;

(ii) $M<\frac{\Delta \sigma^{2}}{\Delta r}$, the mutant becomes extinct with a probability which decreases as $M$ increases.

(d) $\Delta r<0, \Delta \sigma^{2}<0$ :

(i) $M>\frac{\Delta \sigma^{2}}{\Delta r}$, the mutant becomes extinct almost surely;

(ii) $M<\frac{\Delta \sigma^{2}}{\Delta r}$, the mutant invades with a probability which decreases as $M$ increases.

These studies entail that the sign of the function

$s=\Delta r-\frac{1}{M} \Delta \sigma^{2}$

determines the outcome of selection.

\section{Selective dynamics: unlimited and limited resources}

The reciprocal nature of the interaction between organism and environment entails that the outcome of competition between a resident and a variant type will depend on the integration of two processes:

(a) The resource-metabolic process: the relative capacity of the type to acquire the resources which the environment avails.

This property necessarily depends on the metabolic efficiency of the organism and its capacity to transform the free energy of resources into metabolic energy which can be utilized for offspring production.

(b) The metabolic-demographic process: the relative capacity of the type to convert the metabolic energy of the organism into netoffspring production.
The relative contribution of these two factors to the outcome of selection will depend on the extent to which the abundance of resource determines the selective outcome.

If the resource abundance is unlimited, a change $\Delta R$ in resource abundance will have no effect on the net-reproductive function. The outcome of competition will be driven completely by the metabolic-demographic process and differences in the capacity to acquire resource will not constitute a limiting factor in the selection process.

However, when resource abundance is limited, a change $\Delta R$ will induce a change in the net-reproductive function and consequently an alteration in the demographic parameters. The outcome of competition will be determined by the resource-metabolic process and differences in the effect of these processes on the net-reproductive function.

\subsection{Resource abundance unlimited}

The outcome of competition is determined by the metabolicdemographic process. Consequently, the functions $\Delta r$ and $\Delta \sigma^{2}$ will be independent of the resource abundance.

The competitive interaction process described in Section 4.3 indicates that the selective advantage is given by the expression

$s=\Delta r-\frac{1}{M} \Delta \sigma^{2}$.

We observe from this expression that when population size $M \rightarrow \infty$, the measure of selective advantage reduces to

$s=\Delta r$.

\subsection{Resource abundance limited}

The outcome of competition is now contingent on the resourcemetabolic process. The parameters $\Delta r$ and $\Delta \sigma^{2}$ depend on the relative effect of resource constraints on the net-reproductive function of the incumbent and variant.

Assuming that the changes in $V_{j}$ induced by a mutation and a change in resource abundance can be described by functions

$\tilde{V}_{j}=V_{j}^{1+\delta_{1}}, \quad \tilde{V}_{j}^{*}=\tilde{V}_{j}^{1+\delta_{2}}$,

where $\delta_{1}$ and $\delta_{2}$ are small, we conclude that the net-reproductive function of the mutant is given by

$\tilde{V}_{j}^{*}=\tilde{V}_{j}^{1+\delta}$

where $\delta=\delta_{1}+\delta_{2}$.

In view of the perturbation relations, we have (Eq. (13)),

$\Delta r \approx \phi \delta, \quad \Delta \sigma^{2} \approx \gamma \delta$.

We also have

$\Delta H=-\sigma^{2} \delta$.

Write

$\tilde{s}=-\left(\phi-\frac{\gamma}{M}\right) \Delta S$.

Since, Demetrius and Ziehe (2007),

$\Delta H \cdot \Delta S>0$,

we infer

$\tilde{s}>0$.

Hence when resource abundance is limited, the selective advantage is given by 
Table 2

Invasion criteria. (A) Measure of selective advantage according to changes in the Malthusian parameter $r$. (B) Measure of selective advantage according to changes in entropy $S$, and to ecological constraints measured by the reproduction potential $\phi$ and the demographic index $\gamma . M$ is population size; a.s. means almost surely.

\begin{tabular}{|c|c|c|}
\hline \multicolumn{3}{|l|}{ (A) } \\
\hline Selective constraint & \multicolumn{2}{|l|}{ Selective outcome } \\
\hline$\Delta r>0$ & \multirow{2}{*}{\multicolumn{2}{|c|}{$\begin{array}{l}\text { Variant invades } \\
\text { Variant becomes extinct }\end{array}$}} \\
\hline$\Delta r<0$ & & \\
\hline \multicolumn{3}{|l|}{ (B) } \\
\hline Ecological constraint & Selective constraint & Selective outcome \\
\hline$\phi<0, \gamma>0$ & $\begin{array}{l}\Delta S>0 \\
\Delta S<0\end{array}$ & $\begin{array}{l}\text { Invasion occurs a.s. } \\
\text { Extinction occurs a.s. }\end{array}$ \\
\hline$\phi>0, \gamma<0$ & $\begin{array}{l}\Delta S<0 \\
\Delta S>0\end{array}$ & $\begin{array}{l}\text { Invasion occurs a.s. } \\
\text { Extinction occurs a.s. }\end{array}$ \\
\hline $\begin{array}{l}\phi<0, \gamma<0 \\
M>\gamma / \phi \\
M<\gamma / \phi\end{array}$ & $\begin{array}{l}\Delta S>0 \\
\Delta S>0\end{array}$ & $\begin{array}{l}\text { Invasion occurs a.s. } \\
\text { Invasion with a probability } \\
\text { increasing with } M\end{array}$ \\
\hline $\begin{array}{l}\phi>0, \gamma>0 \\
M>\gamma / \phi \\
M<\gamma / \phi\end{array}$ & $\begin{array}{l}\Delta S<0 \\
\Delta S<0\end{array}$ & $\begin{array}{l}\text { Invasion occurs a.s. } \\
\text { Invasion with a probability } \\
\text { increasing with } M\end{array}$ \\
\hline
\end{tabular}

$s=-\left(\phi-\frac{\gamma}{M}\right) \Delta S$

The quantities $\phi$ and $\gamma$ are demographic variables, analytic functions of the age-specific fecundity and mortality variables.

We will now assume that the population process and the environmental process are in dynamic equilibrium. We can therefore infer from the organism-environment interaction principle that the demographic parameters $\phi$ and $\gamma$ will be correlated with the environmental variables resource abundance and resource diversity. Hence, when resource abundance is limited, the outcome of selection is a stochastic process which is predicted by the entropy and contingent on resource abundance and diversity.

Now, when population size $M \rightarrow \infty$, the selective advantage is independent of resource diversity and is given by

$s=-\phi \Delta S$.

The relation between the constraints on $\phi$ and $\gamma$ and the selective outcome is described in Table 2(B).

\section{The relation between demographic parameters and ecologi- cal constraints}

The organism-environment interaction principle indicates a correlation between environmental variables, as expressed by the resource abundance and resource heterogeneity, and the demographic parameters, reproductive potential $\phi$ and demography index $\gamma$. We will exploit the relation and a specification of $\phi$ and $\gamma$ in terms of other demographic variables in order to specify more sharply the relation between resource variation and demographic parameters.

(a) The relation between the reproductive potential and resource constraints. The reproductive potential $\phi$ is given by $\phi=r-H$. We observe that $\phi<0$ entails that the population growth rate is less than the rate $H$ at which the population returns to its steady state after a random perturbation. In view of the assumed correlation between changes in growth rate and changes in resource abundance, and between changes in the stability of resources and changes in the stability of population numbers, we conclude that $\phi<0$ corresponds to a constant limited resource. A similar argument applies to the condition $\phi>0$. We infer that $\phi>0$ corresponds to resources that undergo large variation in abundance.
Table 3

Demographic parameters $\phi, \gamma$ and their correspondence to the resource distribution.

\begin{tabular}{lll}
\hline & $\gamma>0$ & $\gamma<0$ \\
\hline$\phi<0$ & Limited, stable, & Limited, stable, \\
& heterogeneous composition & homogeneous composition \\
$\phi>0$ & Abundant, unstable, & Abundant, unstable, \\
& heterogeneous composition & homogeneous composition \\
\hline
\end{tabular}

(b) The relation between the demographic index and resource constraints. The demographic index $\gamma$ is given by $\gamma=2 \sigma^{2}+$ $\kappa$. We now observe from (17) that $\gamma<0$ entails that the demographic variance $\sigma^{2}$ is less than the skewness, measured by $\kappa$. As $\sigma^{2}$ measures the dispersion of the net-reproductive function, if we assume a positive correlation between resource diversity and demographic variance, we conclude that $\gamma<$ 0 corresponds to a homogeneous resource distribution. A similar argument indicates that $\gamma>0$ characterizes a heterogeneous resource distribution-one defined by a highly diverse resource.

Table 3 summarizes the relation between the demographic variables $\phi$ and $\gamma$ and the resource constrains derived from the above qualitative argument.

\subsection{Numerical analysis}

The qualitative argument for the characterization of $\phi$ and $\gamma$ in terms of the resource constraints implicitly assumes certain range of values for the parameter $\phi$ and $\gamma$. These values are determined by the net-reproductive function $V(x)$. We will investigate this range of values by a numerical study of $\phi$ and $\gamma$ for certain canonical forms of the net-reproductive function.

We construct a synthetic net-reproductive function $V(x)$ whose shape is parameterized by flatness, skewness and peak value, this latter parameter being correlated with maximal fecundity. The shape parameters are varied, and the corresponding values of the reproductive potential $\phi$ and demographic index $\gamma$ are computed (Appendix A). The numerical analysis shows that (Appendix A, Fig. A.2):

(1) $\phi$ decreases as the shape of the net-reproductive function goes from left-skewed to right-skewed, whatever the flatness (as schematized in Fig. 2(B)).

(2) $\phi<0$ corresponds to a flat net-reproductive function, independently of the skewness. Moreover, the parameter region where $\phi<0$ enlarges when the peak value (maximal fecundity) decreases. The shape of the net-reproductive function reflects resources that are constant and limited, as in Table 3.

The pattern for the demographic index $\gamma$ is more complex (Appendix A, Fig. A.3). The parameter region where $\gamma$ is negative is small, delimited by a narrow range of intermediate flatness values, with skewness ranging from no skewness to right skewness. Over the narrow range of flatness $\gamma$ becomes strongly negative. Over a narrow range of flatness adjacent to the previous one, $\gamma$ becomes strongly positive. Outside these regions, $\gamma$ is small and positive. This pattern holds true whatever the peak value. To summarize:

- The condition $\gamma<0$ is rare.

- The condition $\gamma<0$ shows up when the net-reproductive function is moderately flat and right-skewed (as schematized in Fig. 2(C)).

- There exists a region where a small change in the shape of the net-reproductive function can produce an abrupt change in $\gamma$.

This latter point will not be discussed in this study. 
Table 4

Demographic variables for Daphnia pulex at increasing densities (number of individuals per cc): Malthusian growth rate $r$, generation time $T$ (days), entropy $H$, reproductive potential $\phi$, demographic index $\gamma$. Source: Data from Frank et al. (1957).

\begin{tabular}{ccllll}
\hline Population density & $r$ & $T$ & $H$ & $\phi$ & $\gamma$ \\
\hline 1 & 0.2936 & 10.74 & 0.2244 & 0.069 & 0.064 \\
2 & 0.2774 & 11.13 & 0.2253 & 0.052 & 0.23 \\
4 & 0.2683 & 11.23 & 0.2281 & 0.040 & 0.37 \\
8 & 0.1924 & 14.21 & 0.1927 & -0.0003 & 0.25 \\
16 & 0.094 & 17.14 & 0.1712 & -0.077 & 0.29 \\
32 & -0.114 & 20.4 & 0.1486 & -0.262 & 1.66 \\
\hline
\end{tabular}

\subsection{Empirical data}

We will now appeal to empirical data from life-history studies of three species, Daphnia, Rotifers and Drosophila, to furnish support for the characteristics given in Table 3.

Daphnia. Daphnia are common freshwater micro-crustaceans that are often dominant herbivores in the lakes and ponds they inhabit. The organisms have considerable ecological importance and in view of the facility with which they can be cultured in the laboratory, they have become a popular species in studies of lifehistory evolution.

A significant feature of the organism for ecological and lifehistory studies derives from the fact that the organism undergo cyclical parthenogenetic reproduction. This entails that the effect of environmental and resource constraints on life-history variation can be investigated independent of genetic variation.

The life-history data we analyze is a summary of investigation carried out by Frank et al. (1957). The objective was to study the effect of density on the fecundity and survivorship parameters. Frank et al. (1957) constructed cohort life-tables with a single clone that has been maintained as a lineage for several years.

Daphnia pulex matures when it is about ten days old, producing clutches of various offspring number (1-100 eggs) every day over its 20-80 days life span. The experimental study was based on cohorts from individuals that were less than one day old. Each cohort was placed in $25 \mathrm{ml}$ of synthetic pond water. The experimental set up had seven treatment densities of $1,2,4,8,16$, 24,32 individuals per $\mathrm{cc}$, and produced population sizes ranging from 25 to 800 individuals. The life-table for each density was constructed. We have used these life-tables to compute the various demographic parameters (Table 4, Fig. 4).

The population density represents a good measure of the resource variation in abundance. The graphs in Fig. 4 indicate that $\phi$ decreases with density. At low density, $\phi>0$, at high density, $\phi<0$. The empirical data support the relation between resource abundance and $\phi$. The graph also shows that $\gamma$ is always positive and shows a sharp increase at higher densities. It is not possible to make any direct inference regarding $\gamma$ and resource heterogeneity in this case, unless we assume that resource heterogeneity increases with density in these populations.

Rotifers. The study of Ricci (1983) describes the life-history traits of nine species of Bdelloids rotifers collected in two contrasting environments: bottom samples from water courses and moss samples from trees and walls. The terrestrial environment can be considered stable, and the aquatic environment unstable. Indeed, despite its potential instability, the terrestrial environment provides stable resources in favorable conditions, and rotifers have developed elaborate strategies to cope with unfavorable conditions (anhydrobiosis). By contrast, in the aquatic environment, rotifers are subject to strong predation pressures and resources may fluctuate by the interplay of numerous species at various levels of large trophic networks. This point of view is consistent with the values of the growth rate $r$ and the generation time $T$, which are

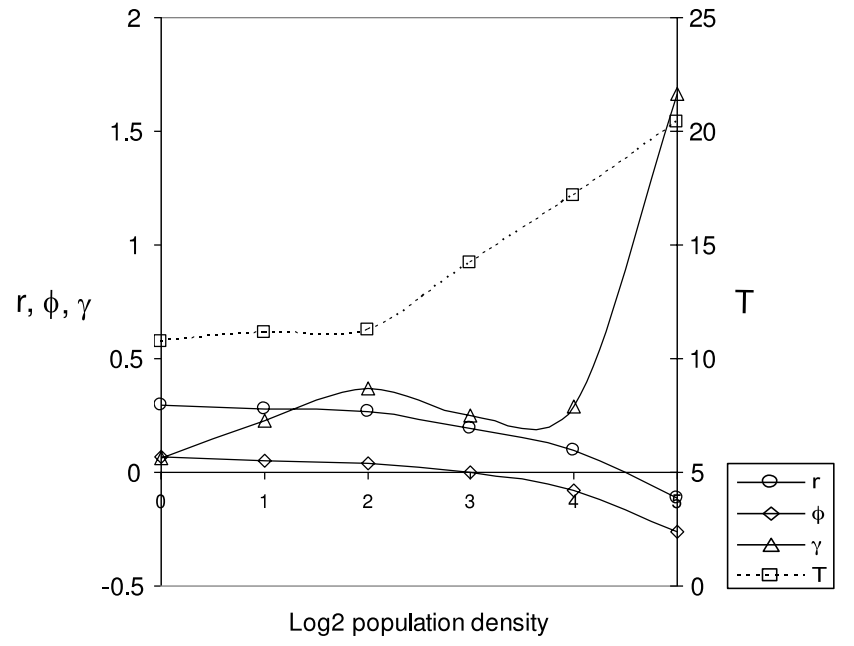

Fig. 4. Demographic parameters for Daphnia populations at increasing population densities: growth rate $r$, generation time $T$, reproductive potential $\phi$, and demographic index $\gamma$. The growth rate $r$ decreases with population density while the generation time $T$ increases. The reproductive potential $\phi$ is positive at low density, and negative at high density.

Source: Data from Frank et al. (1957).

Table 5

Demographic variables for Bdelloid rotifer species in two contrasting environments: $\mathrm{Aq}=$ Aquatic, $\mathrm{Tr}=$ Terrestrial. Malthusian growth rate $r$, generation time $T$ (days), entropy rate $H$, reproductive potential $\phi$, demographic index $\gamma$. Source: Data from Ricci (1983).

\begin{tabular}{lllrlrl}
\hline & Env. & $r$ & \multicolumn{1}{l}{$T$} & \multicolumn{1}{l}{$H$} & \multicolumn{1}{l}{$\phi$} & \multicolumn{1}{l}{$\gamma$} \\
\hline Habrotrocha constricta & $\mathrm{Aq}$ & 0.3386 & 7.93 & 0.2737 & 0.0648 & 0.098 \\
Macrotrachela inermis & $\mathrm{Aq}$ & 0.3291 & 8.13 & 0.2680 & 0.0609 & 0.255 \\
Embata laticeps & $\mathrm{Aq}$ & 0.3118 & 7.49 & 0.3034 & 0.0148 & 0.303 \\
Philodina roseola & $\mathrm{Aq}$ & 0.4264 & 6.80 & 0.3024 & 0.1239 & 0.346 \\
Habrotrocha elusa vegetata & $\mathrm{Tr}$ & 0.2728 & 8.26 & 0.2898 & -0.0170 & 0.496 \\
Habrotrocha sylvestris & $\mathrm{Tr}$ & 0.2749 & 9.10 & 0.2696 & 0.0053 & 0.291 \\
Otostephanos torquatus & $\mathrm{Tr}$ & 0.1060 & 16.25 & 0.1946 & -0.0884 & 0.969 \\
Adineta vaga & $\mathrm{Tr}$ & 0.3713 & 6.26 & 0.2971 & 0.0742 & 0.469 \\
\hline
\end{tabular}

respectively larger and smaller in the aquatic environment than in the terrestrial environment (Table 5, Fig. 5(A)): according to classical life-history theory, the aquatic environment can be associated with $r$-strategist populations and the terrestrial environment with $K$-strategist populations.

The values of $\phi$ and $\gamma$ for the nine rotifer species are displayed in Fig. 5(B)-(C) according to the environment, aquatic (considered unstable and opportunistic) or terrestrial (considered stable and competitive). When going from the aquatic environment to the terrestrial one, the trend of $\phi$ is from positive to negative, and the trend of $\gamma$ is to increase from small positive values to larger positive ones. The values of $\gamma$ are much more dispersed in the terrestrial environment. This could reflect a broader spectrum of ecological niches in this latter environment (Fig. 5(C)). The trends of $\phi$ and $\gamma$ are in agreement with Table 3: the terrestrial environment is characterized by more abundant and more heterogeneous resources.

Drosophila. The study of Thomas-Orillard and Legendre (1996) compares the life-histories of Drosophila melanogaster strains subjected to two constraints: infection by the C-virus, and resource abundance (Appendix B). The Drosophila C virus (DCV) is a non hereditary virus horizontally transmitted to the larvae by consumption of contaminated food. The DCV has pleïotropic effects on its host: it reduces larval survival rates and developmental time but, curiously, enhances the fecundity rates of the contaminated 
A
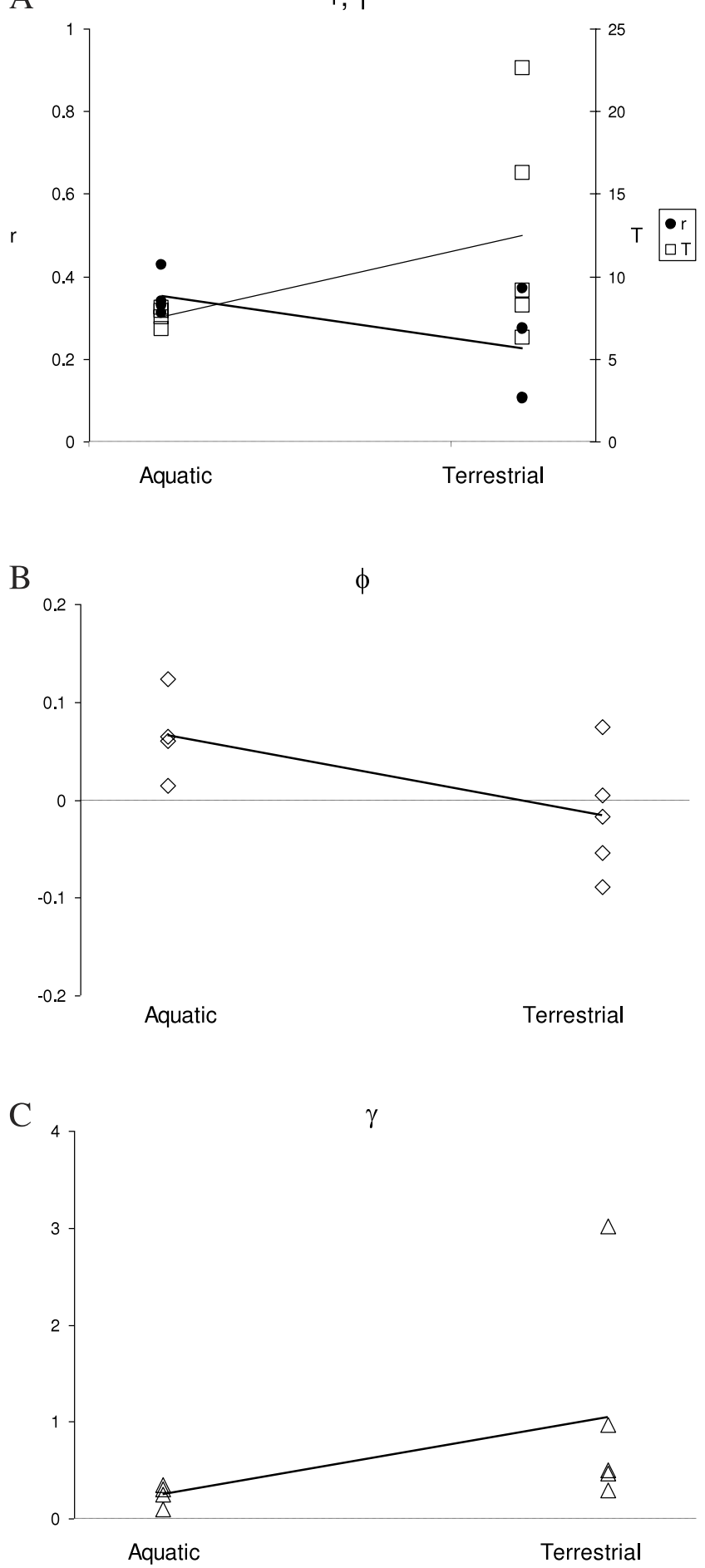

Fig. 5. Demographic parameters for nine species of Bdelloid rotifers in two contrasting environments, aquatic and terrestrial. Regression lines show the trend. (A) Growth rate $r$ and generation time $T$; (B) Reproductive potential $\phi$; (C) Demographic index $\gamma$.

Source: Data from Ricci (1983)

imagos. The net effect is a larger growth rate of the DCV-infected populations.

To better assess the impact of the virus on its host, DCVinfected and DCV-free strains of Drosophila were reared in normal or competitive food conditions, and the demographic parameters determined in each case. Thomas-Orillard and Legendre (1996)

\section{Table 6}

Demographic variables for Drosophila melanogaster, DCV-free and DCV-infected populations in normal and competitive food conditions: Malthusian growth rate $r$, generation time $T$ (days), entropy rate $H$, reproductive potential $\phi$, demographic index $\gamma$.

Source: Data from Thomas-Orillard and Legendre (1996).

\begin{tabular}{lllllll}
\hline & Environment & $r$ & $T$ & $H$ & $\phi$ & $\gamma$ \\
\hline \multirow{2}{*}{ DCV-free } & Normal & 0.3442 & 12.34 & 0.1092 & 0.254 & 0.111 \\
& Competitive & 0.3409 & 12.23 & 0.1106 & 0.230 & 0.040 \\
\hline \multirow{2}{*}{ DCV-infected } & Normal & 0.4057 & 11.06 & 0.1237 & 0.282 & -0.018 \\
& Competitive & 0.3506 & 11.26 & 0.1274 & 0.223 & -0.004 \\
\hline
\end{tabular}

found that the growth rate of DCV-infected animals was still higher in competitive food conditions, though to a lesser extent. However, using a demographic model incorporating competition between the DCV-free and DCV-infected populations, they showed that: (1) if no contamination occurs, the DCV-infected population goes extinct despite its larger growth rate in a density-independent context; (2) if the contamination rate is low, the two populations coexist. It was pointed out that the growth rate is not an absolute measure of fitness, as it does not take the ecological setup into account.

Fig. 6 shows that $\phi>0$ for both the DCV-free and the DCVinfected strains, and that $\phi$ decreases for both strains when going from abundant resource condition to limited resource condition, in accordance with Table 3. For the free individuals, the demographic index $\gamma$ is positive and decreases when going from the normal environment to the competitive one. For the infected individuals, $\gamma$ is negative and does not vary significantly across the two environments (Table 6). The negative value of $\gamma$ accounts for the reduced fitness of the infected individuals, as predicted by the measure of selective advantage in Table 2(B), and in agreement with the result of Thomas-Orillard and Legendre (1996).

\section{Discussion}

Competition between related types for the appropriation of resources and the investment of these resources into the production of offspring constitutes the driving force of evolutionary change by selection.

The classical models of this competition process assert that the outcome of selection is a deterministic process predicted by the Malthusian parameter, the population growth rate $r$. Selective advantage is given by

$s=\Delta r$.

Here $\Delta r$ denotes the difference in growth rate between mutant and incumbent types.

This relation implicitly assumes that population size is infinite and explicitly neglects variations in resource abundance and diversity. When population size is finite, and we consider the variations in abundance and diversity of resources which the populations utilize, then the measure is

$s=-\left(\phi-\frac{\gamma}{M}\right) \Delta S$.

The demographic parameters $\phi$ and $\gamma$ characterize resource abundance and diversity respectively and $M$ denotes population size.

The concept evolutionary entropy describes the diversity in the pathways of energy flow within the population. This is a generic notion which is pertinent to various levels of biological organization: molecular, demographic and ecological (Demetrius et al., 2004). Accordingly the entropic selection principle is applicable to the study of competition between populations at various hierarchical levels: cells, organisms and species. 
A

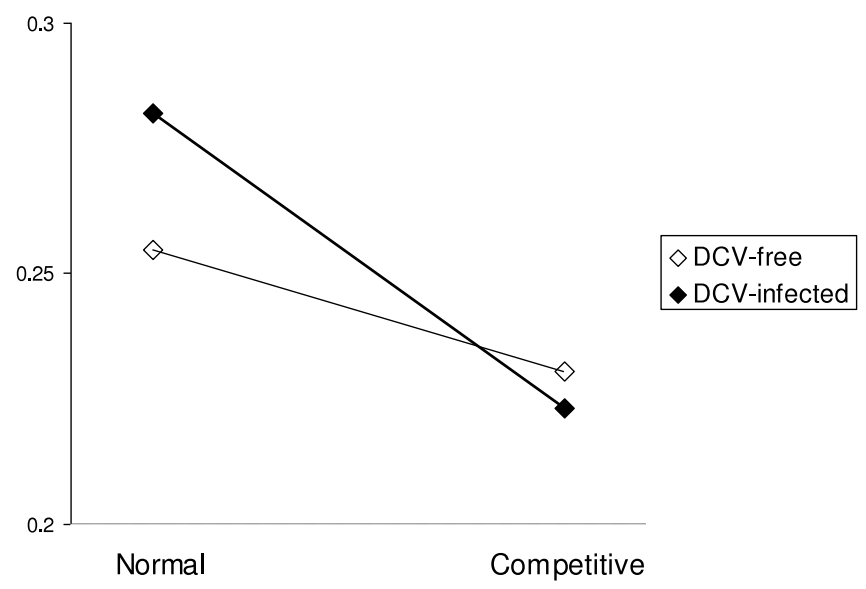

B $\gamma$

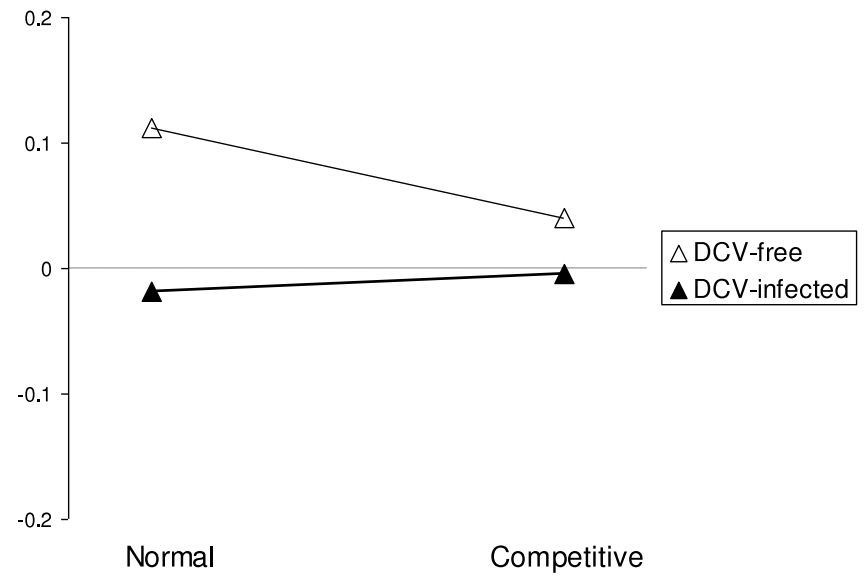

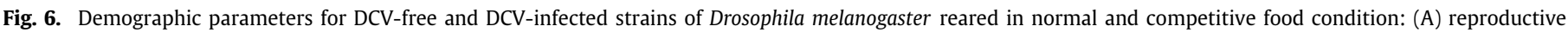
potential $\phi$; (B) demographic index $\gamma$.

Source: Data from Thomas-Orillard and Legendre (1996).

Evolutionary entropy also describes the robustness or stability of the population. Consequently Eq. (22) can be invoked to provide an evolutionary rationale for the large variation in stability observed in biological systems subject to different classes of environmental constraints throughout their evolutionary history.

Variability in the age or size or metabolic energy at which individuals replicate is a fundamental property of all metabolic systems. This variability is observed at the molecular level-as in metabolic networks, at the cellular level-as in the heterogeneity of the cell cycle, and at the population level-as in the iteroparity of life history.

The statistical parameter evolutionary entropy is an analytical description of this heterogeneity. The entropy $S$ is analytically related to generation time $T$. We have

$S=\log T+b$.

This property has been exploited to establish certain analytical relations between entropy and various morphometric and physiological parameters, such as body size, life-span and metabolic rate.
The entropic selection principle established in this article furnishes a new framework for understanding the evolutionary basis for the large variation in body size, life span and metabolic rate observed in natural populations (Demetrius et al., 2009).

\section{Conclusion}

The two fundamental processes that form the core of Darwin's theory of evolution are cooperation between parental alleles in an organism and competition between genetically related types in a population. Mendel's laws of particulate inheritance, which formalize the allelic dynamics of cooperation, and the entropic principle of selection which describes the ecological dynamics of competition, are complementary canons of the Darwinian thesis.

Mendel's laws of heredity postulate that inheritance is particulate, in opposition to the continuous variation observed for many traits. Mendelism, in spite of the empirical support documented by de Vries, Correns and von Tschermak, was considered highly controversial, as it violated the intuitively plausible notion of blending 
parental heritabilities. Mendel's theoretical model only became accepted when Fisher showed that if many genes were involved in the expression of a trait, they could produce the continuous phenotypic variation observed. Fisher's observation can be more formally expressed by the assertion:

The theory of blending inheritance is the limit, as the number of genes involved in the expression of a trait tends to infinity, of the theory of particulate inheritance.

The entropic principle of natural selection asserts that competition between related types is regulated by the stability of population numbers, a property which can be described by evolutionary entropy. Entropic selection is highly consistent with a large range of empirical observations. In particular, the principle is consistent with the well known fact that cancer cells with lower growth rates often outcompete normal cells in microenvironments where the resource is subject to large variations in abundance. The controversial nature of the entropic selection principle resides in the fact that it appears to violate the intuitively plausible notion of Malthusian selection-a mode of selection which seems consistent with laboratory observations.

This article has furnished an explanation for these laboratory observations and has shown that the Malthusian principle and the entropic principle can be reconciled. The rapprochement resides on the following analytical fact:

The Malthusian selection principle is the limit, as population size and resource abundance tend to infinity, of the entropic selection principle.

Consequently, Malthusian selection as proposed by Fisher, is an approximation to the entropic selection principle. The validity of this approximation clearly increases as the population size and resource abundance increase.

The body of work which we call directionality theory is essentially a synthesis of the Mendelian laws of particulate inheritance with the entropic principle of selection. The main thrust of directionality theory is to elucidate how the allelic dynamics of cooperation, as formalized by Mendel's law, are integrated with the ecological dynamics of competition, as described by the entropic selection principle, to predict long-term changes in the genotypic and phenotypic states of a population. The main achievement of this paper in realizing this program is the analytical depiction of the two critical ecological determinants of selection: the resource abundance and its diversity.

\section{Acknowledgment}

We are grateful to Gérard Lacroix for insights on rotifer species.

\section{Appendix A. The incidence of the shape of the net-reproductive function on the reproductive potential $\phi$ and the demographic index $\gamma$}

We construct a synthetic net-reproductive function $V(x)$ whose shape is parameterized by flatness, skewness, and peak value. The shape parameters are varied and the corresponding values of $\phi$ and $\gamma$ are computed. The Gaussian function with mean $a$ and standard deviation $s$,

$g_{s}(x)=\exp \left(-\frac{1}{2} \frac{(x-a)^{2}}{s^{2}}\right)$,

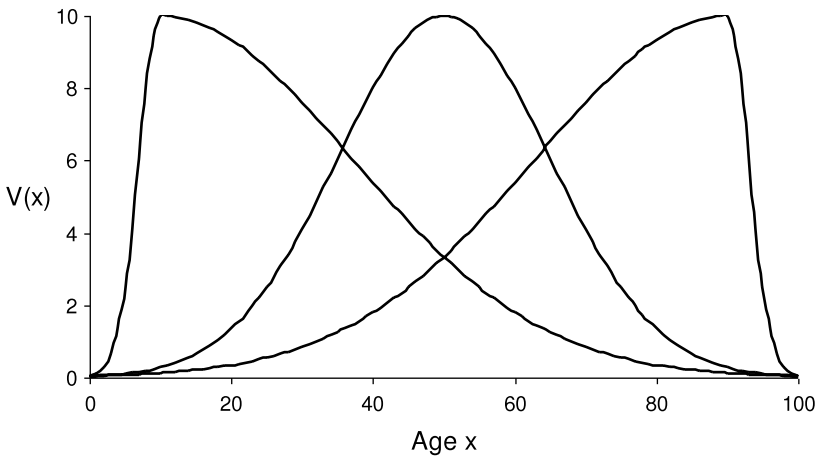

Fig. A.1. The shape of the parameterized net-reproductive function $V_{b, s, f}(x)$. Parameter values: $s=15$ (standard deviation $=$ flatness), $f=10$ (peak value), $b=10,50,90$ (left skewness, no skewness, right skewness).

is made skewed using an homothetic transform of parameter $b$, where the parameter $b$ runs through the age classes, in number $d$ :

$h_{b, s}(x)= \begin{cases}g_{s}\left(\frac{a}{b} x\right), & 1 \leq x \leq b \\ g_{s}\left((d-a) \frac{x-b}{d-b}+a\right), & b<x<d .\end{cases}$

The function $h_{b, s}$ peaks at $x=b$. It is symmetric for $b=a$ (zero skewness), has left skewness for $b<a$, and right skewness for $b>a$. The number $d$ of age classes is fixed, $d=100$, and we take $a=d / 2=50$.

The function $h_{b, s}$ has peak value 1 . The net-reproductive function is now defined as $V_{b, s, f}(x)=f h_{b, s}(x)$ with peak value $f$. The parameter $f$ is correlated with maximal fecundity. Thus the synthetic net-reproductive function $V_{b, s, f}$ is parameterized by $b$ (skewness), $s$ (flatness), and $f$ (peak value) (Fig. A.1). We compute the values of $\phi$ and $\gamma$ associated with $V_{b, s, f}$, and observe how these quantities change when the parameters $b, s, f$ are varied within biologically realistic bounds. For readability of the figures, $b$ and $s$ are varied continuously, and only two typical values of $f(f=10$, $f=2$ ) are given.

\section{Appendix B. Life tables for Drosophila}

\begin{tabular}{|c|c|c|c|c|c|c|c|c|}
\hline \multirow[t]{3}{*}{$\bar{i}$} & \multicolumn{4}{|c|}{$\begin{array}{l}\text { Normal } \\
\text { food-condition }\end{array}$} & \multicolumn{4}{|c|}{$\begin{array}{l}\text { Competitive } \\
\text { food-condition }\end{array}$} \\
\hline & \multicolumn{2}{|c|}{ DCV-free } & \multicolumn{2}{|c|}{$\begin{array}{l}\text { DCV- } \\
\text { infected }\end{array}$} & \multicolumn{2}{|c|}{ DCV-free } & \multicolumn{2}{|c|}{$\begin{array}{l}\text { DCV- } \\
\text { infected }\end{array}$} \\
\hline & $l_{i}$ & $m_{i}$ & $l_{i}$ & $m_{i}$ & $l_{i}$ & $m_{i}$ & $l_{i}$ & $m_{i}$ \\
\hline 1 & 1.000 & 0 & 1.000 & 0 & 1.000 & 0 & 1.000 & 0 \\
\hline 2 & 0.990 & 0 & 0.990 & 0 & 0.990 & 0 & 0.990 & 0 \\
\hline 3 & 0.961 & 0 & 0.923 & 0 & 0.873 & 0 & 0.923 & 0 \\
\hline 4 & 0.933 & 0 & 0.860 & 0 & 0.771 & 0 & 0.860 & 0 \\
\hline 5 & 0.906 & 0 & 0.802 & 0 & 0.680 & 0 & 0.802 & 0 \\
\hline 6 & 0.880 & 0 & 0.747 & 0 & 0.600 & 0 & 0.748 & 0 \\
\hline 7 & 0.878 & 0 & 0.722 & 0 & 0.598 & 0 & 0.722 & 0 \\
\hline 8 & 0.876 & 0 & 0.697 & 0 & 0.597 & 0 & 0.697 & 0 \\
\hline 9 & 0.873 & 0 & 0.673 & 0.9 & 0.595 & 0 & 0.673 & 0.9 \\
\hline 10 & 0.871 & 0 & 0.673 & 29.6 & 0.594 & 0 & 0.673 & 16.2 \\
\hline 11 & 0.871 & 15.00 & 0.673 & 40.0 & 0.594 & 22.3 & 0.673 & 23.3 \\
\hline 12 & 0.871 & 31.00 & 0.673 & 43.2 & 0.594 & 29.9 & 0.673 & 32.8 \\
\hline 13 & 0.871 & 35.00 & 0.673 & 30.5 & 0.594 & 33.7 & 0.673 & 25.4 \\
\hline 14 & 0.871 & 28.70 & & & 0.594 & 30.6 & & \\
\hline
\end{tabular}


A
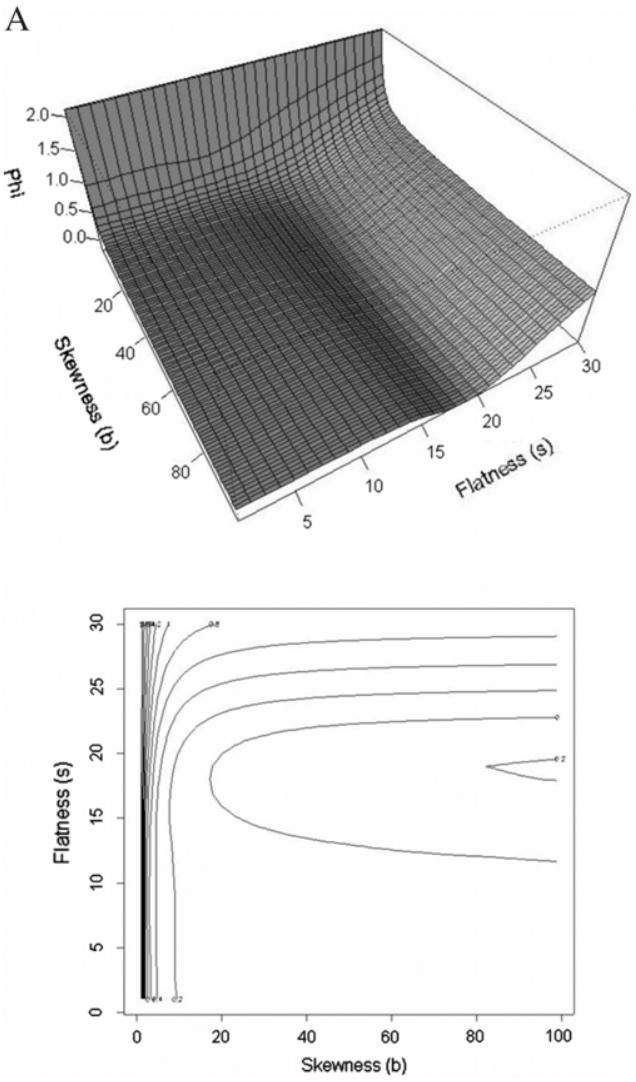

B
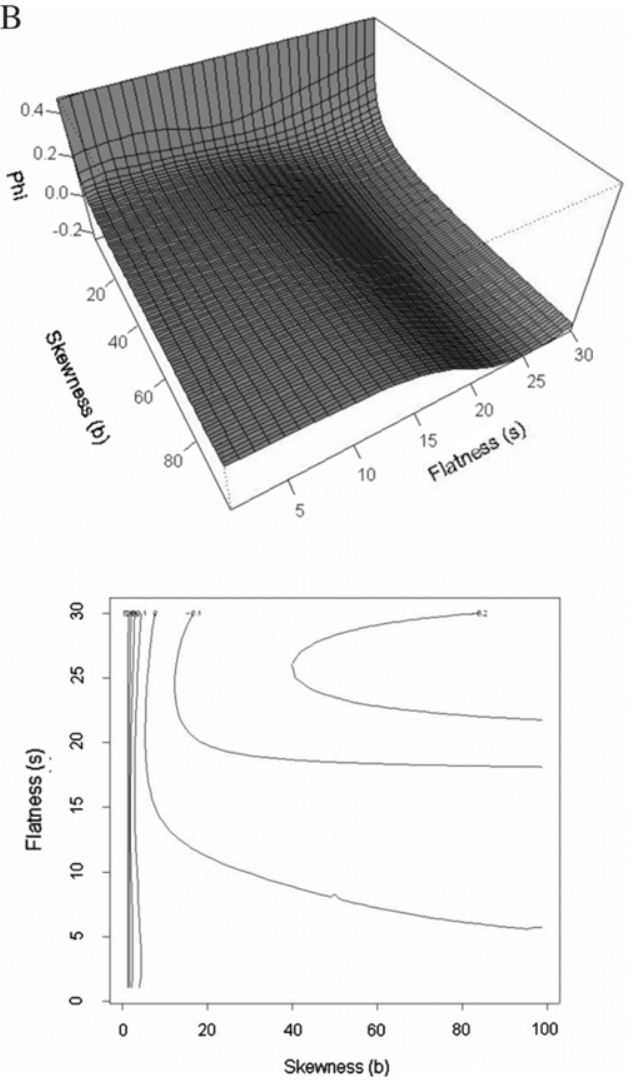

Fig. A.2. Values of the reproductive potential $\phi$ when the shape of the net-reproductive function is varied, parameterized by left skewness to right skewness (no skewness at $b=50$ ), and by increasing flatness. Perspective and contour plot. The contour plot shows the zero-contour line. (A) Peak value $f=10$. (B) Peak value $f=2$.

A
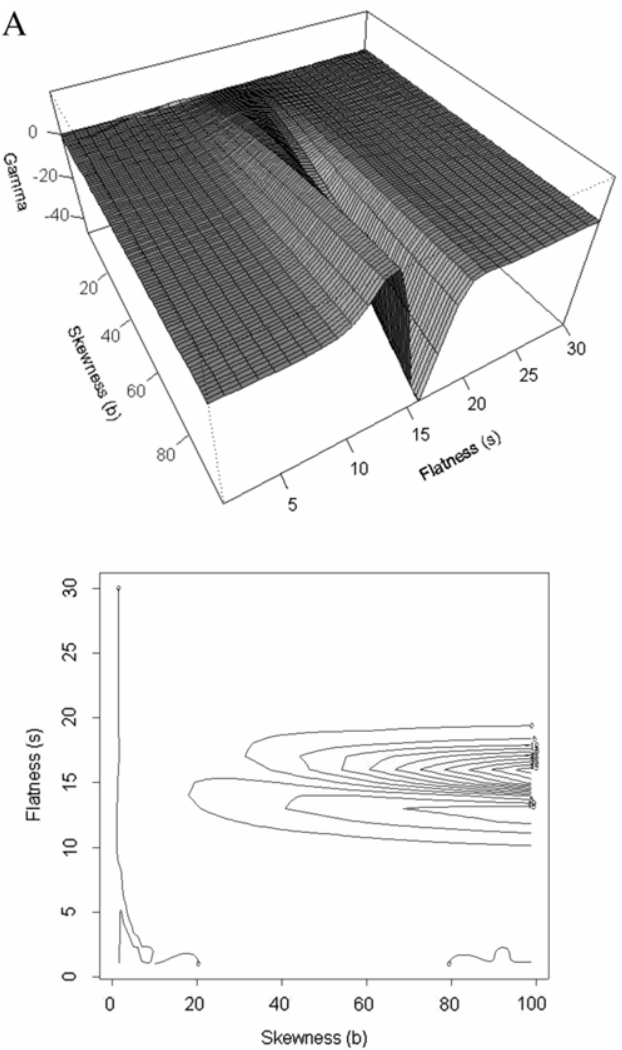

B
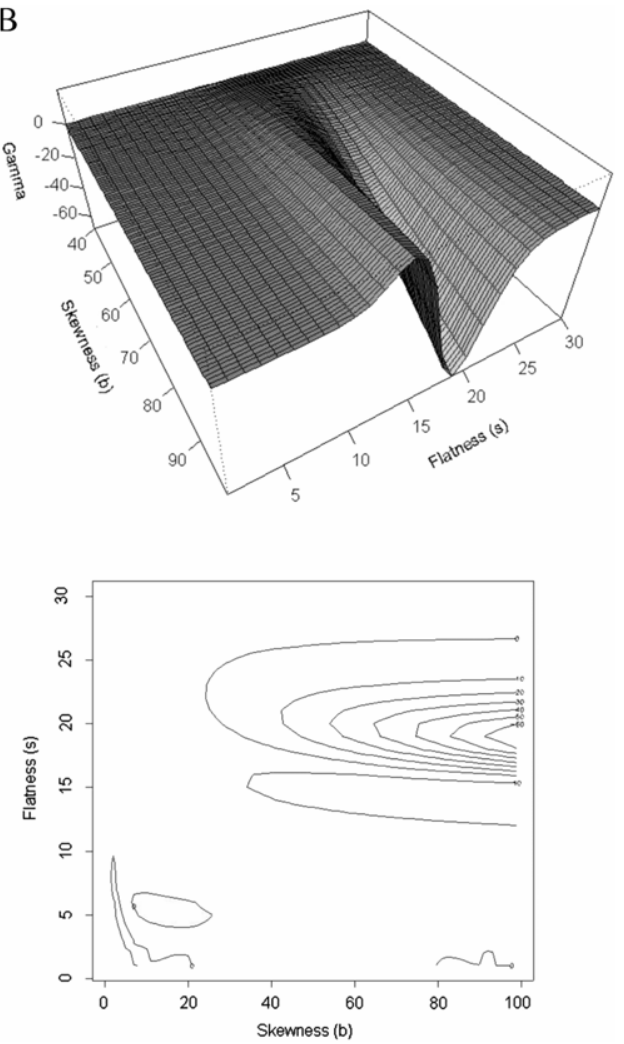

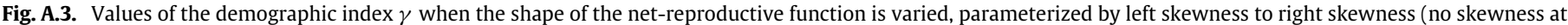
$b=50$ ), and by increasing flatness. Perspective and contour plot. The contour plot shows the zero-contour line. (A) Peak value $f=10$. (B) Peak value $f=2$. 


\section{References}

Charlesworth, B., 1994. The effect of background selection against deleterious mutations on weakly selected, linked variants. Genet. Res. 63, 213-227.

Charlesworth, B., Williamson, J.A., 1975. The probability of survival of a mutant gene in an age-structured population and implication for the evolution of lifehistories. Genet. Res. 26, 1-10.

Crow, J., Kimura, M., 1970. An Introduction to Population Genetic Theory. Harper and Row, New York.

Demetrius, L., 1974. Demographic parameters and natural selection. Proc. Natl. Acad. Sci. USA 71, 4645-4647.

Demetrius, L., 1983. Statistical mechanics and population biology. J. Stat. Phys. 30, 709-753.

Demetrius, L., 1997. Directionality principles in thermodynamics and evolution. Proc. Natl. Acad. Sci. USA 94, 3491-3498.

Demetrius, L., Gundlach, M., 1999. Evolutionary dynamics in random environments, In: Crauel, H., Gundlach, M. (Eds.), Stochastic Dynamics. Springer-Verlag, New York, pp. 371-394.

Demetrius, L., Gundlach, M., Ochs, G., 2004. Complexity and demographic stability in population models. Theor. Popul. Biol. 65, 211-225.

Demetrius, L., Legendre, S., Harremöes, P., 2009. Evolutionary entropy: a predictor of body size, metabolic rate and maximal life span. Bull. Math. Biol. 71, 800-818.

Demetrius, L., Ziehe, M., 2007. Darwinian fitness. Theor. Popul. Biol. 72, 323-345.

Dieckmann, U., Ferrière, R., 2004. Adaptive dynamics and evolving biodiversity. In: Ferrière, R., Dieckmann, U., Couvet, D. (Eds.), Evolutionary Conservation Biology. Cambridge Univ. Press, pp. 188-224.

Ewens, W., 2004. Mathematical Population Genetics. Springer-Verlag. New York.

Fernebro, J., et al., 2008. The influence of in vitro fitness defects on pneumococcal ability to colonize and to cause invasive disease. BMC Microbiol. 8, 65.

Fisher, R.A., 1930. The Genetical Theory of Natural Selection. Oxford Univ. Press, London.

Frank, P.W., Boll, C.D., Kelly, R.W., 1957. Vital statistics of laboratory cultures of Daphnia pulex DeGeer as related to density. Physiol. Zool. 30, 287-305.

Gillespie, J.H., 1974. Natural selection for within generation variance in offspring number. Genetics 76, 601-606.

Hamilton, W.D., 1964. The genetical evolution of social behaviour. I. J. Theoret. Biol. 7, $1-16$.

Hamilton, W.D., 1966. The moulding of senescence by natural selection. J. Theoret. Biol. 12, 12-45.

Houston, A.I., McNamara, J.M., 1999. Models of Adaptive Behavior. Cambridge Univ. Press.
Kisdi, E., Geritz, S.A.H., 1999. Adaptive dynamics in allele space: evolution of genetic polymorphism by small mutations in a heterogeneous environment. Evolution 53, 993-1008.

Kowald, A., Demetrius, L., 2005. Directionality theory: a computational study of an entropic principle in evolutionary processes. Proc. R. Soc. Lond. Ser. B 272, 741-749.

Lawton, B., Brown, W.R., 1986. The population and community ecology of invading insects. Phil. Trans. R. Soc. B 314, 607-617.

Lehmann, L., Keller, L., 2006. The evolution of cooperation and altruism-a general framework and a classification of models. J. Evol. Biol. 19, 1365-1376.

Leturque, H., Rousset, F., 2002. Dispersal, kin competition, and the ideal free distribution in a spatially heterogeneous population. Theor. Popul. Biol. 62 . 169-180.

Levins, R., Lewontin, R., 1985. The Dialectal Biologist. Harvard University Press

Lewontin, R.C., 1982. Organism and environment. In: Plotkin, H. (Ed.), Learning, Development and Culture. John Wiley and Sons, pp. 151-170.

MacArthur, R.H., 1962. Some generalized theorems of natural selection. Proc. Natl. Acad. Sci. USA 48, 1893-1897.

May, R.M., McClean, A.R., Grupta, S., 2001. What characterizes a successful invader? Phil. Trans. R. Soc. B 355, 901-910.

Metz, J.A.J., Nisbet, R.M., Geritz, S.A.H., 1992. How should we define 'fitness' for general ecological scenarios? Trends Ecol. Evol. 7, 198-202.

Rand, D.A., Wilson, H.B., McGlade, J.M., 1994. Dynamics and evolution: evolutionary stable attractors, invasion exponents and phenotype dynamics. Phil. Trans. R. Soc. B 343, 261-283.

Raunkiær, C., 1934. The Life Forms of Plants and Statistical Plant Geography. Oxford University Press, Oxford.

Ricci, C.N., 1983. Life histories of some specie of Rotifera Bdelloidea. Hydrobiologia $104,175-180$.

Roff, D.A., 1992. The Evolution of Life Histories: Theory and Analysis. Chapman and Hall, New York.

Rousset, F., Ronce, O., 2004. Inclusive fitness for traits affecting metapopulation demography. Theor. Popul. Biol. 65, 127-142.

Schmalhausen, I.I., 1949. Factors of Evolution. Blakiston, Philadelphia.

Stearns, S., 1992. The Evolution of Life Histories. Oxford Univ. Press.

Thomas-Orillard, M., Legendre, S., 1996. Virus C de la Drosophile et dynamique d'une population hôte. C. R. Acad. Sci. Paris 319, 615-621.

Wright, S., 1945. The differential equation for the distribution of gene frequency. Proc. Natl. Acad. Sci. USA 31, 382-389.

Ziehe, M., Demetrius, L., 2005. Directionality theory: an empirical study of an entropic principle in life-history evolution. Proc. R. Soc. Lond. Ser. B 272 $1185-1194$. 